\title{
МЕТОДЫ МАТЕМАТИЧЕСКОГО АНАЛИЗА И ПРОГНОЗИРОВАНИЯ ОЦЕНКИ ЗАНЯТОСТИ И ВОЗМОЖНОСТИ ТРУДОУСТРОЙСТВА ВЫПУСКНИКОВ УЧЕБНЫХ ЗАВЕДЕНИЙ
}

\author{
О.В. Баюк, И.О. Лозикова \\ Тюменский индустриальный университет, г. Тюмень
}

Новизна исследования заключается в разработке качественно нового подхода к оценке кадрового потенциала, основанного на методах оценки и прогнозирования персонала.

Целью исследования - формирование аналитики в разрезе различных видов предприятий/отраслей/регионов, что позволяет разрабатывать качественные рекомендации для развития профессиональных кадров в том или ином направлении, для развития социальной и сервисной инфраструктуры в регионе для удержания профессиональных кадров.

Актуальностью работы является структуризация вопроса кадровой подготовки в учебных заведениях и возможности контролирования (прогнозирования) трудоустройства выпускников на предприятиях, как важный элемент человеческого капитала. Актуальность данного вопроса очевидна, в связи с возможностью выявлением количественных показателей и выявление его зависимости от основных факторов влияния.

В работе представлены две математические модели прогнозирования системы занятости. В ней выполнен математический анализ трудоустройства выпускников учебных заведений любого уровня (колледжей, высших учебных заведений, курсов повышения квалификации и прочее), проведено прогнозирование востребованности этих выпускников предприятиями региона/отрасли/страны.

Результатом работы является разработка математического аппарата необходимого для выполнения полноценного анализа и дальнейшее прогнозирования (востребованности) специальности (программы/курса) на рынке труда, как в определённом регионе, так и в стране в целом и построение демографических моделей.

Использование математического аппарата позволит потенциальным пользователям (учебным заведением, работодателям и другим заинтересованным лицам) получить инструмент и количественные показатели для дальнейшего планирования работы при подготовке специалистов и разработке образовательных программ.

Ключевые слова: математическое моделирование, трудоустройство выпускников, оценка и прогнозирование занятости

\section{ВВЕДЕНИЕ}

Характерной особенностью формирования рынка труда в России является дифференцированное его развитие в регионах и отраслях. Такая тенденция объясняется рядом факторов: большой территорией страны, неравномерностью распределения ресурсов, неоднородностью экономической структуры и прочее.

Сегодня Россия для реализации политики индустриализации все больше нуждается в высококвалифицированных рабочих кадрах, которые могли бы соответствовать запросам инновационного развития страны, быть конкурентоспособными. По предварительному анализу рынка труда до 2020 года в России наиболее востребованными станут технические, информационные и профессиональные специальности [1].

Ситуация в сфере трудоустройства для молодежи по-прежнему напряженная. Недостаточный уровень образования, низкая профессиональная подготовка, отсутствие производственного опыта, плохая информированность о состоянии спроса на рынке труда, высокая конкуренция, стихийная трудовая миграция молодого населения и при этом высокие запросы соискателей по заработной плате [2].
Молодежь составляет примерно треть всего населения России. А потому сегодня государство предпринимает ряд мер по обеспечению молодых людей работой [3]. В последнее время количество вакансий на рынке труда увеличилось, и по прогнозам экспертов их число будет и дальше расти. Предприятия вернулись к реализации программ развития персонала, которые временно были приостановлены [4]. Очевидно, что услуги по подбору, развитию, обучению персонала снова актуальны и необходимы. Однако требования к качеству таких услуг становятся все более жесткими. Несмотря на то, что на протяжении последних нескольких лет российские работодатели предпочитают местные кадры, дефицитных специалистов по-прежнему приглашают из-за рубежа. Чаще всего это уникальные специалисты редких профессий и технические руководители [5].

\section{ОСНОВНАЯ ЧАСТЬ}

Для разработки реальной системы прогнозирования социально-экономической ситуации на рынке труда необходимо решить ряд задач. 
Шаг 1. Определим список факторов, влияющих на трудоустройство, $\mathrm{x}=\left(\mathrm{x}_{1}, \mathrm{x}_{2}, \mathrm{x}_{3}, \mathrm{x}_{4}, \ldots \mathrm{x}_{\mathrm{i}}, \ldots \mathrm{x}_{\mathrm{n}}\right)$.

Например,

$x_{1}$ - средняя зарплата;

$x_{2}$ - количество безработных;

$x_{3}$ - количество выпускников;

$x_{4}$ - количество компаний, имеющих вакантные должности по различным отраслям в регионе и/или за его пределами;

$x_{n}$ - другие значимые факторы.

Шаг 2. Обозначим выбранные факторы с учетом предыдущих статистических данных: $\left(x_{1}{ }^{j}, x_{2}{ }^{j}, \ldots, x_{i}^{j}, \ldots, x_{n}{ }^{j}\right)-$ вектор значений выбранных факторов за ј -й год.

Шаг 3. Выполним корреляцию, т.е. определим однофакторную зависимость $r\left(x_{i}, x_{j}\right)$ и тесноту связи выбранных факторов $x_{i}$ и $x_{j}$ друг от друга для всех $i, j=\overline{1, n}$.

Например:

- оценкой качества выпускников работодателями и учебными заведениями;

- средней заработной платой и количеством отработанных лет;

- количеством узконаправленных специалистов и количеством мест на предприятии, принимающих на работу таких специалистов;

- числом выпускников учреждений и количеством устроившихся по специальности и т.п.

Шаг 4. Для оценки не измеряемых факторов (фактических или искусственных) применим следующий способ: каждый специалист при моделировании реального мира (предприятия, вуза, региона и пр.) получает результат, при объединении результатов выполним их анализ и обобщаем результат, делаем общий вывод.

Шаг 5. Для каждого $x_{i}$ фактора найдем зависимость от $x_{j}, j=\overline{1, n}$.

Шаг 6. Далее строим математическую модель в виде системы обыкновенных дифференциальных уравнений [6].

$$
\frac{d x}{d t}=f(u, x, p, t)
$$

где $u$ - управляемые и неуправляемые внешние факторы; мира.

$p$ - факторы, описывающие модель реального

Шаг 7. С помощью математических методов, например, теории подобия с использованием однофакторных и/или многофакторных моделей вычислим значения параметров $p$ [7]. Далее оценим адекватность полученной модели. Если модель адекватна, то продолжим решение, если модель не адекватна, то возвращаемся к шагу 6 , откорректировать данные дифференциальной функции $f(x, t, u, p)$.

Шаг 8. Для нахождения прогнозного значения параметров $\tilde{o}$ в момент времени $\mathrm{t} 1$ воспользуемся методами решения обыкновенных дифференциальных уравнений (Эйлера, Рунге-Кутты, Милна) и определим значение $\tilde{o}$ на отрезке времени $\left[t_{0}, t_{1}\right] n p u$ начальных условиях $t=t_{0} \quad u x\left(t_{0}\right)=x_{0}$.

Далее определим экстремум (прогнозное) значение

$$
g_{i t} \rightarrow \min , i=\overline{1, n}
$$

где $i$ - номер фактора;

$t$ - момент времени.

Для выполнения прогнозной оценки ожидаемого значения параметра можно использовать два подхода с помощью:

а) модели временных рядов (авторегрессии);

б) линейной регрессионной модели.

Модель временных рядов

Индекс $t$ принимаем как временной параметр (ежедневный, квартальный или годовой), тогда функция будет равна:

$$
g_{t}=\sum_{i=1}^{p} \alpha_{i} g_{t-i}, \quad t=\overline{p+1, N}
$$

где $\alpha_{i}, i=\overline{1, p}$ - весовые коэффициенты.

Составим функцию управления с учетом сезонности факторов

$$
J=\sum_{t=p+1}^{N}\left(g_{t}-\sum_{i=1}^{p} \alpha_{i} g_{t-i}\right)^{2}
$$

Численные значения весовых коэффициентов $\alpha_{i}, i=\overline{1, p}$ определим из уравнения (4):

$$
\frac{\partial}{\partial a_{i}} J\left(\alpha_{i}\right)=0 \quad i=\overline{1, p}
$$

Условия минимума функции (5) сводятся к решению СЛАУ. Решая СЛАУ численными методами - например, методами простой итерации или ГауссаЗейделя [8], получим прогнозное значение параметра $g_{N+1}$.

Для определения периодических свойств временного ряда аппроксимируем функцию $y(t)$ :

$$
y(t)=A_{0}+\sum_{j=1}^{v}\left(A_{j} \cos \frac{2 \pi}{T_{j}} t+B_{j} \sin \frac{2 \pi}{T_{j}} t\right)
$$

где $t \in[-L, L], \quad L=\frac{N}{2}, \quad t_{N}=\frac{N}{2} . u z\left(t_{i}\right)=g\left(t_{i}-t_{N}\right)$.

Для определения коэффициентов $A_{0}, A_{j}, B_{j}, T_{j}, j=$ $\overline{1, v}$ воспользуемся формулой:

$$
F\left(A_{0}, A_{j}, B_{j}, T_{j}\right)=\sum_{i=1}^{N}\left(z\left(t_{i}\right)-y\left(t_{i}\right)\right)^{2}
$$

где $N$-число измерений.

Алгоритм нахождения коэффициентов: 
Шаг 1. $A_{0}=\frac{1}{N} \sum_{i=1}^{N} z\left(t_{i}\right)$.

Шаг 2. Определяем $\widehat{\mathrm{z}}\left(t_{i}\right)=z\left(t_{i}\right)-A_{0}$.

Шаг 3. Зададим $j=1$.

Шаг 4. Вычисляем коэффициенты $\tilde{z}\left(t_{i}\right)=\hat{z}\left(t_{i}\right)-$ $\sum_{m=1}^{j-1}\left(A_{m} \cos \frac{2 \pi}{T_{m}} t_{i}+B_{m} \sin \frac{2 \pi}{T_{m}} t_{i}\right)$

Шаг 5. Определим коэффициенты $A_{j}, B_{j}, T_{j}$, из условия экстремума функции

$$
\tilde{F}\left(A_{j}, B_{j}, T_{j}\right)=\sum_{i=1}^{N}\left(\tilde{z}\left(t_{i}\right)-A_{j} \cos \frac{2 \pi}{T_{j}} t_{i}+B_{j} \sin \frac{2 \pi}{T_{j}} t_{i}\right)^{2}
$$

$$
\frac{\partial \tilde{F}\left(A_{j}, B_{j}, T_{j}\right)}{\partial A_{j}}=0 \quad \frac{\partial \tilde{F}\left(A_{j}, B_{j}, T_{j}\right)}{\partial B_{j}}=0 \quad \frac{\partial \tilde{F}\left(A_{j}, B_{j}, T_{j}\right)}{\partial T_{j}}=0
$$

Получим систему нелинейных уравнений (8).

Обозначим

$$
\begin{aligned}
& p_{1}=\sum_{i=1}^{N} \tilde{z}\left(t_{i}\right) \cos \frac{2 \pi}{T_{j}} t_{i} ; \\
& p_{2}=\sum_{i=1}^{N} \tilde{z}\left(t_{i}\right) \sin \frac{2 \pi}{T_{j}} t_{i} ; \\
& q_{1}=\sum_{i=1}^{N} \tilde{z}\left(t_{i}\right)\left(\cos \frac{2 \pi}{T_{j}} t_{i}\right)^{2} \\
& q_{1}=\sum_{i=1}^{N} \tilde{z}\left(t_{i}\right)\left(\sin \frac{2 \pi}{T_{j}} t_{i}\right)^{2} \\
& q_{2}=\sum_{i=1}^{N} \tilde{z}\left(t_{i}\right) \cos \frac{2 \pi}{T_{j}} t_{i} \sin \frac{2 \pi}{T_{j}} \mathrm{t}_{\mathrm{i}} \\
& \Delta=q_{1} q_{3}-q_{2}^{2} .
\end{aligned}
$$

Получим

$$
A_{j}=\left(p_{1} q_{3}-p_{2} q_{2}\right) / \Delta \quad B_{j}=\left(p_{2} q_{1}-p_{1} q_{2}\right) / \Delta \quad \text { (9) }
$$

Путем подстановки в функцию $\tilde{F}\left(A_{j}, B_{j}, T_{j}\right)$ выражения (9) получаем функцию

$$
\widehat{F}\left(T_{j}\right)=\sum_{i=1}^{N}\left(z\left(t_{i}\right)-f\left(T_{j}\right)\right)^{2}
$$

где $f\left(T_{j}\right)=A_{j}\left(T_{j}\right) \cos \frac{2 \pi}{T_{j}} t_{i}+B_{j}\left(T_{j}\right) \sin \frac{2 \pi}{T_{j}} t_{i}$. (10).

Методом градиента определим минимум функции

Шаг 6. Далее определяем коэффициенты $A_{j}, B_{j}, T_{j}$ по формуле (9).

Шаг 7. Вычисляется значение функции

$$
\begin{aligned}
& F_{j}=\sum_{m=1}^{N}\left(z\left(t_{i}\right)-A_{0}\right. \\
& \left.-\sum_{m=1}^{j}\left(A_{m} \cos \frac{2 \pi}{T_{m}} t_{i}+B_{m} \sin \frac{2 \pi}{T_{m}} t_{i}\right)\right)^{2}
\end{aligned}
$$

Если $a b s\left(F_{j}-F_{j-1}\right)<\varepsilon$, то переходим к шагу 8, иначе к шагу 4.

Шаг 8. Далее определяем число итераций $j$ и коэффициенты $A_{0}, A_{m}, B_{m}, T_{m}, m=\overline{1, j}$.

Определим значение $g_{t}$ :

$$
g_{t}=\sum_{i=1}^{q} \beta_{i} g_{t-i * T} \quad t=\overline{q * T, N}
$$

Здесь, коэффициенты влияния $\beta_{i}, i=\overline{1, p}$, вычислим аналогично (4)

Функция управления равна

$$
J=\sum_{t=p+1}^{N}\left(g_{t}-\sum_{i=1}^{q} \beta_{i} g_{t-i * T}\right)^{2}
$$

Сводя решение к решению СЛАУ $q$-го порядка методами Гаусса-Зейделя или простой итерации вычислим коэффициенты влияния $\beta_{i}, i=\overline{1, q}$ и отсюда получим прогнозные значения параметра $g_{N+1}$ :

$$
g_{N+1}=\sum_{i=1}^{q} \beta_{i} g_{N+1-i * T}
$$

Обозначим $p$ через $q$ получим следующую модель:

$$
g_{t}=\sum_{i=1}^{p} \alpha_{i} g_{t-i}+\sum_{j=1}^{q} \beta_{j} g_{t-j * T},
$$

где $t=\overline{\max (p+1, q * T), N}$

Весовые коэффициенты $\alpha_{i}, i=\overline{1, p} \quad$ и $\quad \beta_{i}, i=$ $\overline{1, q}$ получим из условия экстремума функции

$$
J=\sum_{t=p+1}^{N}\left(g_{t}-\sum_{i=1}^{p} \alpha_{i} g_{t-i}-\sum_{j=1}^{q} \beta_{j} g_{t-j * T}\right)^{2}
$$

Таким образом, прогнозное значение с помощью модели временных рядов определим по формуле:

$$
g_{N+1}=\sum_{i=1}^{p} \alpha_{i} g_{N+1-i}-\sum_{j=1}^{q} \beta_{j} g_{N+1-j * T}
$$

\section{Линейная регрессионная модель}

Для определения регрессионной зависимости выберем те же исходные данные, что и для задачи выше, $\quad g_{i j}, i=\overline{1, n} j=1, \ldots$ где $m-$ это число выбранных параметров $(1 \leq m \leq n)$.

Алгоритм решения:

Шаг 1. Для всех $n$ параметров вычисляется корреляционную матрицу $q_{i j}$, матрица состоит из $n$ строк и $n$ столбцов элементы $\mathrm{q}_{\mathrm{ij}}$ вычисляются по формуле:

$$
q_{i j}=\frac{\sum_{k=1}^{N}\left(g_{i k}-\overline{g_{l}}\right)\left(g_{j k}-\overline{g_{l}}\right)}{\sqrt{\sum_{k=1}^{N}\left(g_{i k}-\overline{g_{l}}\right) \sum_{k=1}^{N}\left(g_{i k}-\overline{g_{l}}\right)}} i=\overline{1, n}, j=\overline{1, n}
$$

где $N$ - число данных по каждому выбранному параметру, $\quad \bar{q}_{l}, i=\overline{1, n}$, - среднее значение $i$-го параметра. 
Шаг 2. Для каждого і-го параметра выберем $\operatorname{abs}\left(q_{i j}\right) \rightarrow \max , j=\overline{1, n}$

Шаг 3. Получим следующую регрессионную модель

$$
y_{t+1}=\sum_{i=1}^{m} \alpha_{i} x_{i t}, t=\overline{1, N}
$$

где $\alpha_{i}, i=\overline{1, m}$ весовые коэффициенты.

Шаг 4. Получим функцию управления

$$
J=\left(\sum_{t=1}^{N-1} y_{t+1}-\sum_{i=1}^{m} \alpha_{i} x_{i t}\right)^{2}
$$

Коэффициенты $\alpha_{i}, \quad i=\overline{1, m}$ определим по аналогии с формулами (4):

$$
\frac{\partial}{\partial \alpha_{i}} J\left(\alpha_{i}\right)=0, i=\overline{1, m}
$$

Таким образом, прогнозное значение регрессионной модели определим по формуле:

$$
y_{N+1}=\sum_{i=1}^{m} \alpha_{i} x_{i N}
$$

\section{ЗАКЛЮЧЕНИЕ}

Решая математическую модель одним из способов можно спрогнозировать значение $\mathrm{g}_{\mathrm{N}+1}$ или $\mathrm{y}_{\mathrm{N}+1}$ в момент времени $t+1$.

Две методики математической прогнозной оценки выпускников учебных заведений позволят всестороннее изучить кадровые вопросы на предприятиях региона, базирующейся на методологии оценки временных рядов и регрессионных зависимостях $[9,10]$.

Получение количественных показателей дает возможность своевременно вносить изменения, проводить годовой прогноз востребованности выпускников в различных областях (добавлять или заменять специальности), совместно корректировать общеобразовательные программы и тем самым повышать качественный уровень подготовки специалистов $[11,12]$.

\section{СПИСОК ИСПОЛЬЗОВАННЫХ ИСТОЧНИКОВ}

1. Рынок труда и востребованных профессий в 2020-2021 годы в России: анализ и статистика. 2021// [Электрон. ресурс] - URL: https://visasam.ru/russia/ rabotavrf/rynok-truda-v-rossii.html (дата обращения 20.03.2021)

2. К вопросу оценки эффективности функционирования системы управления персоналом. 2018 // [Электронный ресурс] URL: https://articlekz.com (дата обращения 07.04.2021)

3. МОШУРЕНКО П.С. Актуальные вопросы развития инновационной экономики. 2017//Вестник НИЦ МИСИ: Актуальные вопросы современной науки, ООО "Научный инновационный центр Международный институт стратегических исследований" (Москва). - №2: 21-33.

4. Хохлова М.Г. 2019. Молодежный рынок труда: европейский опыт в российском контексте//Мировая экономика и международные отношения, Национальный исследовательский институт мировой экономики и международных отношений им. Е.М. Примакова РАН, Российская академия наук (Москва):118-128.

5. Климова Н. В., Михеева В.А. 2016. Проблемы молодежной занятости в контексте несоответствия рынка образовательных услуг и рынка труда// Политематический сетевой электронный научный журнал Кубанского государственного аграрного университета. Кубанский государственный аграрный университет им. И.Т. Трубилина (Краснодар): 1289-1304.

6. Шахоростова О.Н. 2014. Дифференциация причин дефицита трудовых ресурсов: региональный аспект //Молодой ученый, ООО "Издательство Молодой ученый" (Казань): 336-338.

7. Третьякова Л.А. 2015. Особенности развития рынка труда как основного элемента устойчивого экономического развития территорий//Инновации в АПК: проблемы и перспективы, Белгородский государственный аграрный университет имени В.Я. Горина (Майский): 33-46.

8. Баюк О.В. 2015. Создание информационно-образовательной среды системы дистанционного обучения// материалы XI Международной научно-практической конференции: в 2-х частях: 50-54.

9. Абдразаковна Д.Ш., Мазаков Т.Ж., Жайдарова А.М. 2015. Система обеспечения безопасности и «Демография»//Евразийское научное объединение: 4-7.

10. Баюк О.В. 2016. Оптимизация блочно-модульной структуры информационного базиса адаптивно-обучающих систем// The Europe and world Science, Engineering and Techology, Materials of the international scientific-practical conference. Editorial Board: Chairman of the Board S. Midelski:131-138.

11. Маулитов А.Е., Ашимханова Д.Э. 2017. Эффективность реализации государственной молодежной политики в Республики Казахстан//Вестник Киргизско-Российский славянский университет (Бишкек): 66-70.

12 Бондаренко А.Н., Югфельд А.С. 2013. Управление рынком труда/Учебное пособие, Тулький филиал РАНХиГС: 67.

Баюк Ольга Васильевна - дочент кафедры Кибернетических систем, Тюменского индустриального университета института геологии и нефтегазодобычи, ТИУ ИГиН, .тел. (3254),283018, e-mail: bayuk_o@mail.ru.

Лозикова Инна Олеговна - старший преподаватель кафедры Кибернетических систем, Тюменского индустриального университета института геологии и нефтегазодобычи, ТИУ ИГиН,. тел.(3254),283018, e-mail: lozikovaio@tyiui.ru 


\title{
METHODS OF MATHEMATICAL ANALYSIS AND FORECASTING OF EMPLOYMENT ASSESSMENT AND EMPLOYMENT OPPORTUNITIES FOR GRADUATES OF EDUCATIONAL INSTITUTIONS
}

\author{
O. V. Bayuk, I.O. Lozikova \\ Tyumen Industrial University, Tyumen
}

The novelty of the research lies in the development of a qualitatively new approach to the assessment of human resources potential, based on the methods of personnel assessment and forecasting.

The purpose of the study is to form analytics in the context of various types of enterprises/industries/regions, which allows us to develop high-quality recommendations for the development of professional personnel in a particular direction, for the development of social and service infrastructure in the region to retain professional personnel.

The relevance of the work is the structuring of the issue of personnel training in educational institutions and the possibility of monitoring (forecasting) the employment of graduates in enterprises, as an important element of human capital.

The relevance of this issue is obvious, due to the possibility of identifying quantitative indicators and identifying its dependence on the main factors of influence.

The paper presents two mathematical models for predicting the employment system, it performs a mathematical analysis

Index terms: mathematical modeling, employment of graduates, assessment and forecasting of employment

\section{REFERENCES}

1. The labor market and professions in demand in 2020-2021 in Russia: analysis and statistics. 2021// [Electron. resource] - URL: https://visasam.ru/russia/rabotavrf/rynok-truda-v-rossii.html (date of treatment 03/20/2021) (in Russian)

2. On the issue of assessing the effectiveness of the functioning of the personnel management system. 2018// [Electronic resource] - URL: https://articlekz.com (date of access 07.04.2021) (in Russian)

3. MOSHURENKO P.S. 2017. Topical issues of development of an innovative economy// Bulletin of the Research Center IISS: Topical issues of modern science, Scientific Innovation Center International Institute for Strategic Research (Moscow). - No. 2:21-33. (in Russian)

4. Khokhlova M.G. 2019. Youth labor market: European experience in the Russian context// World Economy and International Relations, National Research Institute of World Economy and International Relations. EAT. Primakov RAS, Russian Academy of Sciences (Moscow):118-128. (in Russian)

5. Klimova N.V., Mikheeva V.A. 2016. Problems of youth employment in the context of the mismatch between the educational services market and the labor market // Polythematic network electronic scientific journal of the Kuban State Agrarian University. - Kuban State Agrarian University named after I.T. Trubilin (Krasnodar): 1289-1304. (in Russian)

6. Shakhorostova O.N. 2014. Differentiation of the reasons for the shortage of labor resources: the regional aspect // Young Scientist, LLC "Young Scientist Publishing House" (Kazan): 336-338. (in Russian)

7. L.A. Tretyakova, 2015. Features of the development of the labor market as the main element of sustainable economic development of territories // Innovations in the agro-industrial complex: problems and prospects, Belgorod State Agrarian University named after V.Ya. Gorin (Maisky): 33-46. (in Russian)

8. Bayuk O.V. 2015. Creation of an information and educational environment for a distance learning system // materials of the XI International Scientific and Practical Conference: in 2 parts: 50-54. (in Russian)

9. Abdrazakovna D.Sh., Mazakov T.Zh., Zhaidarova A.M. 2015. Security system and "Demography" // Eurasian Scientific Association: 4-7. (in Russian)

10. Bayuk O.V. 2016. Optimization of the block-modular structure of the information basis of adaptive learning systems // The Europe and world Science, Engineering and Techology, Materials of the international scientific-practical conference. Editorial Board: Chairman of the Board S. Midelski: 131-138. (in Russian)

11. Maulitov A.E., Ashimkhanova D.E. 2017. The effectiveness of the implementation of state youth policy in the Republic of Kazakhstan // Bulletin of the Kyrgyz-Russian Slavic University (Bishkek): 66-70. (in Russian)

12. Bondarenko A.N., Yugfeld A.S. 2013. Labor market management / Textbook, Tulkiy branch of RANEPA: 67. (in Russian)

Bayuk Olga Vasilyevna - Associate Professor of the Department of Cybernetic Systems, Tyumen Industrial University, Institute of Geology and Oil and Gas Production, TIU IGiN,. tel. (3254),283018, e-mail: bayuk_o@mail.ru.

Inna Lozikova - Senior Lecturer of the Department of Cybernetic Systems, Tyumen Industrial University, Institute of Geology and Oil and Gas Production, TIU IGiN, tel. (3254),283018, e-mail: lozikovaio@tyiui.ru 